\title{
A note on the Poljak-Rödl function
}

\author{
Xuding Zhu* \\ Department of Mathematics \\ Zhejiang Normal University \\ Jinhua, Zhejiang, China \\ xdzhu@zjnu.edu.cn
}

Submitted: Feb 16, 2020; Accepted: Jun 22, 2020; Published: Jul 10, 2020

(c) The author. Released under the CC BY-ND license (International 4.0).

\begin{abstract}
The Poljak-Rödl function is defined as $f(c)=\min \{\chi(G \times H): \chi(G)=\chi(H)=$ $c\}$. This note proves that $\lim \sup _{c \rightarrow \infty} \frac{f(c)}{c} \leqslant \frac{1}{2}$.
\end{abstract}

Mathematics Subject Classifications: 05C88, 05C89

The categorical product (also known as the tensor product or the direct product) $G \times H$ of $G$ and $H$ has vertex set

$$
V(G \times H)=\{(x, y): x \in V(G), y \in V(H)\}
$$

and edge set

$$
E(G \times H)=\left\{(x, y)\left(x^{\prime}, y^{\prime}\right): x x^{\prime} \in E(G), y y^{\prime} \in E(H)\right\} .
$$

The Poljak-Rödl function [8] is defined as

$$
f(c)=\min \{\chi(G \times H): \chi(G)=\chi(H)=c\} .
$$

A proper colouring $\phi$ of $G$ induces a proper colouring $\Phi$ of $G \times H$ defined as $\Phi(x, y)=$ $\phi(x)$. So $\chi(G \times H) \leqslant \chi(G)$. Therefore $f(c) \leqslant c$ for all positive integers $c$. Hedetniemi conjectured in 1966 [4] that $f(c)=c$ for all positive integers $c$. This conjecture received a lot of attention $[1,5,9,12,14,15]$ and was confirmed for $c \leqslant 4[1]$. However the conjecture was disproved by Shitov in [10] in 2019. For a positive integer $c$, let $[c]=\{1,2, \ldots, c\}$. For a graph $G$, the exponential graph $K_{c}^{G}$ has vertex set

$$
V\left(K_{c}^{G}\right)=\{f: f \text { is a mapping } V(G) \rightarrow[c]\}
$$

and in which $f$ and $g$ are adjacent if and only if for any edge $x y$ of $G, f(x) \neq g(y)$. For any graph $G$ and any positive integer $c$, the mapping $\phi: V\left(G \times K_{c}^{G}\right) \rightarrow[c]$ defined as

\footnotetext{
*Supported by NSFC: 11971438.
} 
$\phi(x, f)=f(x)$ is a proper $c$-colouring of $G \times K_{c}^{G}$. So $\chi\left(G \times K_{c}^{G}\right) \leqslant c$. On the other hand, if $\phi$ is a proper $c$-colouring of $G \times H$, then the mapping sending $u \in V(H)$ to $f_{u} \in V\left(K_{c}^{G}\right)$ defined as $f_{u}(v)=\phi(v, u)$ is a homomorphism from $H$ to $K_{c}^{G}$ and hence $\chi(H) \leqslant \chi\left(K_{c}^{G}\right)$. Thus Hedetniemi's conjecture is equivalent to the statement that "if $\chi(G)>c$, then $K_{c}^{G}$ is $c$-colourable."

The lexicographic product $G\left[K_{q}\right]$ of $G$ and $K_{q}$ is the graph obtained from $G$ by replacing each vertex of $G$ with a $q$-clique. Thus $G\left[K_{q}\right]$ has vertex set $\left\{(x, i): x \in V(G), i \in V\left(K_{q}\right)\right\}$ and $(x, i) \sim(y, j)$ if $x \sim y$ or $x=y$ and $i \neq j$. The fractional chromatic number of $G$ is defined as

$$
\chi_{f}(G)=\inf \left\{\frac{\chi\left(G\left[K_{q}\right]\right)}{q}: q \in \mathbb{N}\right\} .
$$

Shitov disproved Hedetniemi's conjecture by showing the following result:

Theorem 1 (Shitov). Assume $|V(G)|=p, \chi_{f}(G) \geqslant 3.1$, $\operatorname{girth}(G) \geqslant 6, q$ is sufficiently large and $c=\lceil 3.1 q\rceil$. Then $\chi\left(G\left[K_{q}\right]\right)>c$ and $\chi\left(K_{c}^{G\left[K_{q}\right]}\right)>c$.

As a consequence of this result, we have $f(c)<c$ for sufficiently large $c$. Using Shitov's result, Tardif and Zhu [13] showed that for sufficiently large $c, f(c) \leqslant c-(\log c)^{1 / 4-o(1)}$. Tardif and Zhu also asked if there is a constant $\epsilon>0$ such that $f(c) \leqslant(1-\epsilon) c$ for sufficiently large $c$ and showed that if a special case of Stahl's conjecture in [11] on the multi-chromatic number of Kneser graphs is true, then $\limsup _{c \rightarrow \infty} f(c) / c \leqslant 1 / 2$. The above question was answered by He and Wigderson [3], who showed that for $\epsilon \approx 10^{-9}$, $f(c) \leqslant(1-\epsilon) c$ for sufficiently large $c$.

On the other hand, the problem whether $f(c)$ is bounded by a constant remains a challenging open problem. It is known $[7,8,14]$ that $f(c)$ is either bounded by 9 to goes to infinity.

This note shows that $\lim \sup _{c \rightarrow \infty} f(c) / c \leqslant 1 / 2$ without assuming Stahl's conjecture.

Theorem 2. For $d \geqslant 1$, let $G$ be a graph of girth 6 and with $\chi_{f}(G) \geqslant 6.3 d$. Let $p=|V(G)|, q$ is sufficiently large and $c=\lceil 3.1 q\rceil$. Then $\chi\left(G\left[K_{q}\right]\right) \geqslant 2 d c-2 c+2$ and $\chi\left(K_{d c}^{G\left[K_{q}\right]}\right) \geqslant 2 d c-2 c+2$. Consequently, $f(2 d c-2 c+2) \leqslant d c$.

Proof. It is well-known that $\chi\left(G\left[K_{q}\right]\right) \geqslant \chi_{f}(G) q \geqslant 6.3 d q \geqslant 2 d c>2 d c-2 c+2$. Now we show that $\chi\left(K_{d c}^{G\left[K_{q}\right]}\right) \geqslant 2 d c-2 c+2$.

Assume $\Psi$ is a $(d c+t)$-colouring of $K_{d c}^{G\left[K_{q}\right]}$ with colour set $[d c+t]$. We shall show that $d c+t \geqslant 2 d c-2 c+2$, i.e., $t \geqslant d c-2 c+2$. Let $S=[d c+t] \backslash[d c]$. The colours in $[d c]$ are called primary colours and colours in $S$ are called secondary colours.

For $i \in[d c]$, denote by $g_{i} \in V\left(K_{c}^{G\left[K_{q}\right]}\right)$ the constant map $g_{i}((x, j))=i$ for all $(x, j) \in$ $G\left[K_{q}\right]$. The set $\left\{g_{i}: i \in[d c]\right\}$ induces a $d c$-clique in $K_{d c}^{G\left[K_{q}\right]}$. Thus we may assume that $\Psi\left(g_{i}\right)=i$ for $i \in[d c]$.

For any $\phi \in V\left(K_{d c}^{G\left[K_{q}\right]}\right)$, let $\operatorname{Im}(\phi)=\left\{\phi(x, i):(x, i) \in V\left(G\left[K_{q}\right]\right)\right\}$ be the image set of $\phi$. If $i \notin \operatorname{Im}(\phi)$, then $\phi \sim g_{i}$. Hence $\Psi(\phi) \neq \Psi\left(g_{i}\right)=i$. Thus for any $\phi \in V\left(K_{d c}^{G\left[K_{q}\right]}\right)$, $\Psi(\phi) \in \operatorname{Im}(\phi) \cup S$. 
For positive integers $m \geqslant 2 k$, let $K(m, k)$ be the Kneser graph whose vertices are $k$-susbets of $[m]$, and for two $k$-subsets $A, B$ of $[m], A \sim B$ if $A \cap B=\emptyset$. It was proved by Lovász in [6] that $\chi(K(m, k))=m-2 k+2$.

For a $c$-subset $A$ of $[c d]$, let $H_{A}$ be the subgraph of $K_{c d}^{G\left[K_{q}\right]}$ induced by

$$
\left\{\phi \in V\left(K_{c d}^{G\left[K_{q}\right]}\right): \operatorname{Im}(\phi) \subseteq A\right\} .
$$

Then $H_{A}$ is isomorphic to $K_{c}^{G\left[K_{q}\right]}$. By Theorem $1,\left|\Psi\left(H_{A}\right)\right| \geqslant c+1$. For every $\phi \in V\left(H_{A}\right)$, we have $\operatorname{Im}(\phi) \subseteq A$. Since $\left|\Psi\left(H_{A}\right)\right| \geqslant c+1>|A|, \Psi\left(H_{A}\right)$ contains at least one secondary colour. Let $\tau(A)$ be an arbitrary secondary colour contained in $\Psi\left(H_{A}\right)$.

If $A, B$ are $c$-subsets of $[d c]$ and $A \cap B=\emptyset$, then every vertex in $H_{A}$ is adjacent to every vertex in $H_{B}$. Hence $\Psi\left(H_{A}\right) \cap \Psi\left(H_{B}\right)=\emptyset$. In particular, $\tau(A) \neq \tau(B)$. Thus $\tau$ is a proper colouring of the Kneser graph $K(d c, c)$. As $\chi(K(d c, c))=d c-2 c+2$, we conclude that $t=|S| \geqslant d c-2 c+2$. This completes the proof of Theorem 2 .

For a positive integer $d$, let $p=p(d)$ be the minimum number of vertices of a graph $G$ with girth 6 and $\chi_{f}(G) \geqslant 6.2 d$. It follows from Theorem 2 that for any sufficiently large integer $q$ (which depends on $p), f(2(d-1) \times\lceil 3.1 q\rceil+2) \leqslant\lceil 3.1 q\rceil d$. As $f(c)$ is nondecreasing, for integers $c$ in the interval $[2(d-1) \times\lceil 3.1 q\rceil+3,2(d-1) \times\lceil 3.1(q+1)\rceil+2]$, we have $f(c) \leqslant\lceil 3.1(q+1)\rceil d$.

Hence for all integers $c \geqslant 2 \times\lceil 3.1 q\rceil(d-1)+2$,

$$
\frac{f(c)}{c} \leqslant \frac{\lceil 3.1(q+1)\rceil d}{2(d-1) \times\lceil 3.1 q\rceil+2} \text {. }
$$

Note that if $d \rightarrow \infty$, then $p=p(d)$ goes to infinity, and hence $q$ goes to infinity. Hence

$$
\limsup _{c \rightarrow \infty} \frac{f(c)}{c} \leqslant \frac{1}{2}
$$

Remark The number $q$ in Theorem 1 is required to be large enough so that some inequalities in the proof are satisified. A careful analysis of these inequalities shows that $q \geqslant 3^{p} p^{3}$ is enough. Let $p$ be the minimum number of vertices of a graph of girth 6 and fractional chromatic number 3.1. The exact value of $p$ is also unknown. A recent computer search by Exoo [2] found a graph on 83 vertices which has odd girth 7 and fractional chromatic number greater than 3.07 (which is enough for Shitov's proof). For $p=83$, we have $c \geqslant 3^{96}$. So the graphs in Theorem 1 have huge chromatic number. Recently, a relatively small counterexample to Hedetniemi's conjecture was constructed in [16]. It is now known that Hedetniemi's conjecture fails for $c \geqslant 125$. Two graphs $G$ and $H$ were constructed in [16], such that $\chi(G), \chi(H)>125$ and $\chi(G \times H) \leqslant 125$, and $G$ and $H$ have 3,403 and 10,501 vertices respectively.

\section{References}

[1] M. El-Zahar and N.W. Sauer, The chromatic number of the product of two 4chromatic graphs is 4, Combinatorica 5 (1985) 121-126. 
[2] G. Exoo, personal communication, 2020.

[3] X. He and Y. Wigderson, Hedetniemi's conjecture is asympototically false, J. Combin. Theory, Ser. B, https://doi.org/10.1016/j.jctb.2020.03.003.

[4] S. Hedetniemi, Homomorphisms of graphs and automata, Technical Report 0310544-T, University of Michigan, 1966.

[5] S. Klavžar, Coloring graph products - a survey, Discrete Math. 155 (1996) 135-145.

[6] L. Lovász, Kneser's conjecture, chromatic number, and homotopy, J. Combin. Theory Ser. A 25 (1978), 319-324.

[7] S. Poljak, Coloring digraphs by iterated antichains, Comment. Math. Univ. Carolin. 32(1991), 209-212.

[8] S. Poljak and V. Rödl, On the arc-chromatic number of a digraph, J. Combin. Theory Ser. B 31(1981), 339-350.

[9] N. Sauer, Hedetniemi's conjecture - a survey, Discrete Math. 229 (2001) 261-292.

[10] Y. Shitov, Counterexamples to Hedetniemi's Conjecture, Ann. of Math. (2) 190(2019), no. 2, 663-667.

[11] S. Stahl, n-tuple colorings and associated graphs, J. Combin. Theory Ser. B 20 (1976), no. 2, 185-203.

[12] C. Tardif, Hedetniemi's conjecture, 40 years later, Graph Theory Notes N. Y. 54 (2008) 46-57.

[13] C. Tardif and X. Zhu, A note on Hedetniemi's conjecture, Stahl's conjecture and the Poljak-Rödl function, Electron. J. Combin. 26(4)(2019), \#P4.32.

[14] X. Zhu, A survey on Hedetniemi's conjecture, Taiwanese J. Math. 2 (1998) 1-24.

[15] X. Zhu, The fractional version of Hedetniemi's conjecture is true, European J. Combin. 32 (2011), 1168-1175.

[16] X. Zhu, Relatively small counterexamples to Hedetniemi's conjecture, arXiv:2004.09028. 